\title{
Epicardial Fat Thickness in Children with Classic Congenital Adrenal Hyperplasia
}

\author{
(1) Kotb Abbass Metwalley ${ }^{1}$, (1) Hekma Saad Farghaly ${ }^{1}$, (1) Abdelrahman Abdelhamid² \\ ${ }^{1}$ Assiut University Faculty of Medicine, Department of Pediatrics, Assiut, Egypt \\ 2South Valley University Qena Faculty of Medicine, Department of Clinical Pathology, Qena, Egypt
}

\section{What is already known on this topic?}

There is an increased risk for cardiac abnormalities in children with congenital adrenal hyperplasia. Epicardial fat thickness is an emerging cardio-metabolic risk factor and has been shown to be related to atherosclerosis.

\section{What this study adds?}

To our knowledge, this is the first study assessing epicardial fat thickness (EFT) in children with congenital adrenal hyperplasia (CAH). EFT is higher in children with CAH than in healthy children and correlated with carotid intima media thickness, left ventricular mass and mitral deceleration time. EFT may be used as a possible marker of early atherosclerosis and myocardial function in children with CAH.

\section{Abstract}

Objective: Epicardial fat thickness (EFT) is an emerging cardio-metabolic risk factor and has been shown to be related to atherosclerosis. EFT has not been studied in the context of CAH. This study aimed to evaluate EFT in children with CAH and its relation to carotid artery intima-media thickness (CA-IMT) and left ventricular (LV) functions.

Methods: Thirty-six children with classical CAH were compared with 36 healthy controls. All patients had confirmed CAH and were receiving steroid substitution therapy. Patients and controls underwent anthropometric evaluation, measurement of fasting lipids, glucose, insulin, homeostasis model assessment for insulin resistance (HOMA-IR). LV functions and EFT were assessed using conventional echocardiography. Duplex ultrasonography was used to measure CA-IMT.

Results: Compared to controls, patients had greater EFT $(p=0.001)$, CA-IMT $(p=0.01)$, LV mass index (LVMI) ( $p=0.001)$ and prolonged mitral deceleration time (DcT) $(p=0.01)$. CAH patients also had significantly worse HOMA-IR $(p=0.001)$ than controls. Abnormalities were worse in uncontrolled $\mathrm{CAH}$ on treatment. Multivariate analysis in $\mathrm{CAH}$ subjects showed EFT correlated positively with waist circumference odds ratio $(\mathrm{OR})[\mathrm{OR}=1.9 ; 95 \%$ confidence interval $(\mathrm{CI}): 1.07-1.14 ; \mathrm{p}=0.01]$, 17-hydroxyprogesterone [OR =1.6; 95\% CI: 1.33-2.89; $\mathrm{p}=0.05]$, testosterone concentration $(\mathrm{OR}=1.7 ; 95 \% \mathrm{CI}: 1.55-2.13 ; \mathrm{p}=0.01)$, LVMI $(\mathrm{OR}=1.14 ; 95 \% \mathrm{Cl}: 1.08-1.13 ; \mathrm{p}=0.01)$, mitral DCT $(O R=2.25 ; 95 \%$ CI: 1.15-2.05; $p=0.01)$ and CA-IMT $(O R=1.6 ; 95 \%$ CI: 1.15-2.05; $p=0.01)$.

Conclusion: EFT is elevated in children with classical CAH, particularly in those with poor control, and is correlated with CA-IMT, LV mass and mitral DcT. Measurement of EFT in CAH children may help to identify those at high risk of developing LV dysfunction and subclinical atherosclerosis.

Keywords: Diastolic function, echocardiography, epicardial fat thickness, left ventricular function, left ventricular mass index, congenital adrenal hyperplasia, carotid intima media thickness, mitral deceleration time

\section{Introduction}

Congenital adrenal hyperplasia (CAH) is an autosomal recessive condition resulting from mutations in enzymes required for adrenal steroid synthesis (1). Defects in the enzyme 21-hydroxylase, leading to enzyme deficiency, are responsible for approximately $95 \%$ of cases (2). CAH is commonly divided into the severe classical and the milder nonclassical form. Classical $\mathrm{CAH}$ is generally subdivided, depending on the extent of enzymatic impairment, into the salt-wasting (SW) form, presenting with both cortisol 
and aldosterone deficiency and the simple virilizing (SV) form, characterized by an isolated cortisol deficiency. Both conditions are associated with androgen excess resulting in virilization of female external genitalia (3). Researchers have long thought that patients with 21-hydroxylase deficiency are at increased risk for cardiovascular diseases due to the resulting high plasma levels of androgens and/or the harmful effects of glucocorticoid and mineralocorticoid treatment (4). 21 -hydroxylase deficiency may also have detrimental effects on vascular structures as well as ventricular systolic and diastolic function (5). Obesity, hypertension, dyslipidaemia and insulin resistance (IR) have been found to be associated with both CAH itself and the treatment strategies (6).

Few studies have utilized carotid artery intima-media thickness (CA-IMT) to assess vascular structural changes in children with $\mathrm{CAH}(4,5,7)$. A hindrance to the wider use of CA-IMT measurements in the pediatric population is the lack of standardization of CA-IMT values in this age group (8). Epicardial fat thickness (EFT) is a layer of adipose tissue surrounding the heart and coronary vessels which can be measured by ultrasound, a simple, noninvasive procedure (9). EFT is a reliable and sensitive marker of cardiovascular risk and has become an emerging target for therapeutic and medical interventions (10). We are not aware of any published data on EFT in children with CAH.

The aim of this study was to evaluate the EFT measurement and its relation to CA-IMT and left ventricular function in a cohort of children with classical CAH.

\section{Methods}

\section{Patients and Methods}

This cross-sectional, controlled study included 36 children (11 males and 25 females; mean \pm standard deviation age $=13.7 \pm 2.4$ years) with a confirmed diagnosis of classic $\mathrm{CAH}$ (4). Diagnosis was made based on clinical signs and biochemical assessment [elevated adrenocorticotropic hormone (ACTH), 17-hydroxyprogesterone (17-OHP), androstenedione and testosterone, in addition to low cortisol]. SW was diagnosed in patients with frank hyponatraemia and hyperkalaemia accompanied by low plasma aldosterone and elevated rennin concentrations (11). Patients were included if they were on glucocorticoid therapy for a minimum of five years. They were recruited during the period between January and December 2017 from the Pediatric Endocrinology Unit of Assiut University Children Hospital, Assiut, Egypt. Patients who had chest deformities, chronic lung disease, poor echo window, pericardial and/ or pleural effusion on transthoracic echocardiography were excluded. Thirty six healthy children ( 7 males and 27 females) matched for age, gender, pubertal status and socioeconomic status were recruited as control subjects from the General Pediatric Outpatient Clinic of the same hospital. None of the controls were hypertensive and none were smokers, on any medication, or had a chronic illness. Controls were attending the outpatient clinic either because of minor illness or accompanying their sick siblings. All patients had classical CAH with 21-hydroxylase deficiency ( $\mathrm{SW} n=30$; SV $n=6)$ and were receiving glucocorticoid substitution therapy with hydrocortisone $(\mathrm{HC})(\mathrm{n}=30)$ or prednisone (PR) $(n=6)$. PR dose was converted to HC using the conversion assumption that $20 \mathrm{mg}$ of $\mathrm{HC}$ is equivalent to $5 \mathrm{mg}$ of PR (12). SW patients were also on 9-alphafludrocortisone therapy at a dose of $50-100 \mathrm{ug} / \mathrm{m}^{2} /$ day. The adequacy of steroid therapy was monitored periodically during follow-up visits every 3-6 months by clinical parameters, such as signs of androgen excess, growth curves, bone age and hormonal assay (13). Patients were divided into two groups according to the degree of control on medical treatment, that is those patients with acceptable disease control and children with poor disease control, based on the previously mentioned data (14).

The study protocol was approved by the Local Ethics Committee of Assiut University Children Hospital, Assiut, Egypt (approval number: 312/2017) and also by the Ethics Committee of the Faculty of Medicine, in accordance to the Declaration of Helsinki. Written informed consent was obtained from the parents of all participants.

All patients and controls were subjected to a full medical history-taking as well as a thorough clinical examination. Demographic and clinical data included age, gender, duration of treatment, type and dose of steroids, blood pressure (BP), height, weight and body mass index (BMI). Systolic BP (SBP) and diastolic BP (DBP) were measured in all subjects in the right arm with a standard sphygmomanometer (Exacta) by the same operator. Height and weight were measured using a wall-mounted stadiometer (seca 213 l) and a calibrated weight scale (Uline Industrial Platform Scales), with the child wearing underwear only. BMI was calculated using the following formula: $\mathrm{BMI}=$ weight $(\mathrm{kg}) /$ height $(\mathrm{m})^{2}$. BMI was expressed as standard deviation scores (SDS) using the Egyptian Growth Reference Data (15). Waist circumference was measured at the midpoint between the lower edge of the ribs in the midaxillary line and the top of the iliac crest by the same clinician. Waist-to-height ratio was then calculated as an index of visceral adiposity. Pubertal status was assessed according to Tanner staging (16). A radiograph of the left hand was used to determine BA using the Greulich-Pyle method (17). This assessment was made in a blinded fashion by a single pediatric endocrinologist. BA 
was defined as advanced when greater than the subject's chronological age by one year or more (18).

Blood samples were drawn after an overnight fast for at least 12 hours at 8.00-10.00 a.m. before the first dose of steroids for assessment of serum levels of total cholesterol (TC), triglycerides (TG), high density lipoprotein-cholesterol (HDL-C), low density lipoprotein cholesterol (LDL-C), glucose and insulin. Serum TG and TC were assessed by quantitative enzymatic colorimetric technique (Bio MerieuxDiagnostic Chemicals Ltd., Charlottetown, CA, USA). Serum high-density lipoproteins (HDL) were measured by the phosphotungstate precipitation method (Biomerieux kit, Marcy L'etoile, Craponne, France). LDL cholesterol was calculated by Friedewald's formula: (TC) - (HDL-C) - 1/5 (TG) (19). IR was calculated using the homeostasis model assessment for IR (HOMA-IR) equation formula:

HOMA-IR = Fasting insulin $(\mathrm{uU} / \mathrm{mL})$ multiplied by fasting glucose $(\mathrm{mmol} / \mathrm{L})$ divided by 22.5 .

A cut-off level of 2.7 was used for diagnosing IR as previously described (20). ACTH, plasma 17-OHP, serum cortisol, androstenedione and testosterone were also measured with commercially available RIA kits (Siemens Healthcare Diagnostics Inc., Los Angeles, CA, USA). The plasma level of high sensitivity $\mathrm{C}$-reactive protein (hSCRP) was measured using the hsCRP enzyme immunoassay test (ELISA) kit for quantitative determination of the CRP concentration in human serum (catalog no. E29-056; Immunospec Corp., Canoga Park, CA, USA).

\section{Echocardiographic Examination}

All echocardiographic examinations were performed according to the recommendations of the American Society of Echocardiography (21). A Philips Envisor Ultrasound System with a S4-2 Broadband Sector (Philips Medical Systems, Inc., Netherlands) was used. Measurements were performed using the machine's incorporated analysis package. An M-mode echocardiography was obtained at the left sternal border. Left ventricular (LV) dimension, LV fractional shortening (FS) and LV ejection fraction (EF) were measured. LV mass index (LVMI) was measured using a LVMI calculator. LV diastolic function was evaluated by mitral inflow velocities obtained in the apical four-chamber view. Mitral filling was assessed with the peak velocity of the transmitral early filling wave (E) and the peak velocity of atrial late filling (A) and the ratio of both (E/A) was calculated. The interval from the early peak velocity to the zero intercept of the extrapolated deceleration time (DcT) slope (early filling mitral DcT) was measured. The interval between the end of the LV outflow velocity and the onset of mitral inflow [isovolumic relaxation time (IVRT)] obtained by pulsed-wave Doppler with the cursor placed in the LV out-flow near the anterior leaflet of the mitral valve, was measured from the end of the LV ejection to the onset of the mitral inflow.

\section{Epicardial Fat Thickness Measurement}

A two-dimensional (2D) echocardiogram, using a standardised procedure, was performed with the patient in the left lateral decubitus position. EFT thickness was measured by an experienced pediatric echocardiologist, who was blinded to the subjects' clinical and demographic data, using the procedure validated by Iacobellis et al (9). EFT was identified as the echolucent region between the external wall of the myocardium and the visceral layer of the pericardium (Figure 1). This thickness was measured perpendicularly on the free wall of the right ventricle at the end of systole over three cardiac cycles, using a parasternal long and a parasternal short axis. The average value of the three cardiac cycles from each echocardiographic view was used for the statistical analysis.

\section{Carotid Intima Media Thickness Measurement}

All participants underwent an ultrasound scan to measure CA-IMT. The studies were performed in the morning between 7:30 and 9:30 a.m. after the children had fasted overnight. All ultrasound scans were performed by an experienced vascular operator who was unaware of the subject's clinical details. Examination of CA-IMT was manually performed using a color duplex flow imaging system (Acuson $128 \mathrm{XP}$; Acuson Corporation, Mountain View, CA, USA). The examinations were performed while the patients were in a supine position, with their necks slightly extended and their heads turned 450 away from the examination side. From both sides of the head, three images were obtained from the distal common carotid

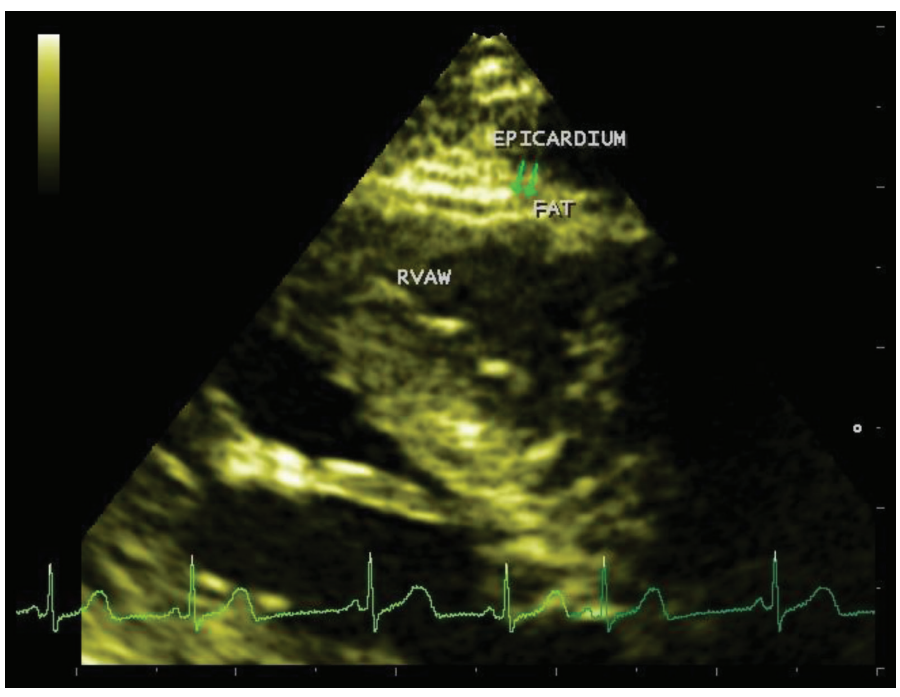

Figure 1. Echocardiographic imaging of the epicardial fat 
artery, 1-2 $\mathrm{cm}$ proximal to the carotid bulb at end diastole. These images were then stored for later offline analyses. All studies were done according to a predetermined, standardized scanning protocol for the right and left carotid arteries (22). All measurements were performed in all participants by the same pediatric cardiologist who was blinded to the clinical and treatment status of the study participants. Reliability of echocardiographic measurements of CA-IMT and EFT were assessed by intra-observer correlation coefficient in all subjects.

\section{Statistical Analysis}

Statistical analysis was performed using the Statistical Package for Social Sciences (SPSS) for Windows, version 16.0 (SPSS Inc, Chicago, IL, USA). Data were expressed as means + standard deviation. Comparisons of quantitative variables between the study groups were made using the paired Student t-test. Correlations between EFT and demographic, clinical, and laboratory variables were assessed using Pearson test. Multiple logistic regression analysis was used to determine the factors that were significantly associated with high EFT. The odds ratios, $95 \%$ confidence intervals and significances were calculated. For all tests, values of $p<0.05$ were considered statistically significant.

\section{Results}

Demographic and anthropometric data of the patients and controls are shown in Table 1. Bone age in the patient group was advanced by an average of two years compared with chronological age. Compared with healthy controls, children with CAH exhibited increased visceral adiposity, as suggested by higher values of BMI SDS, waist circumference, hip circumference and waist to height ratio. Moreover, $\mathrm{CAH}$ children had higher SBP and DBP, although all children had blood pressures within the normal ranges.
Laboratory data of the patients and controls are shown in Table 2. Concentrations of TC, TG, LDL-C, fasting blood glucose, fasting insulin, hsCRP, 17-OHP, androstenedione and testosterone were significantly higher while concentration of HDL-C was significantly lower in $\mathrm{CAH}$ patients compared to control subjects. HOMA-IR values were also significantly higher in the patients compared with controls.

The results of echocardiographic EFT and CA-IMT examinations are shown in Table 3. There were no significant differences in EF and FS values between patients and control subjects. However, compared to control subjects, patients had higher LVMI value, indicating myocardial hypertrophy, and lower E/A ratio, higher IVRT values and prolonged mitral DCT, indicating impaired diastolic function and increased CA-IMT and higher EFT. Intra-observer agreement on CAIMT and EFT measurements were excellent. Intra-observer correlation coefficient was 0.94 and 0.95 , respectively, indicating excellent reproducibility of these measures. Compared to patients who were well controlled $(n=16)$, patients who were uncontrolled $(n=20)$ were older, had advanced bone ages and had higher levels of 17-OHP, testosterone and hsCRP. In addition poorly controlled patients had higher values of LVMI, mitral DcT, EFT and CAIMT (see Table 4).

EFT thickness showed a statistically significant positive correlation with BMI, waist circumference, SBP, DBP, HOMAIR, hsCRP, 17-OHP, testosterone, LVMI, mitral DCT and CAIMT (see Table 5).

Multivariate analysis in children with CAH revealed that EFT was significantly correlated with waist circumference, 17 OHP, HOMA-IR, testosterone, mitral DcT, CA-IMT and LVMI (see Table 6)

Table 1. Demographic, anthropometric and clinical data of the congenital adrenal hyperplasia patients compared with controls

\begin{tabular}{llll}
\hline & CAH patients $(\mathrm{n}=36)$ & Controls $(\mathrm{n}=36)$ & $\mathrm{p}$ value \\
\hline Age, years & $13.7 \pm 2.4$ & $13.6 \pm 2.5$ & $\mathrm{NS}$ \\
SDS-BMI & $1.02 \pm 0.92$ & $-0.24 \pm 1.5$ & 0.01 \\
Waist circumference, cm & $83 \pm 13$ & $72 \pm 14$ & 0.01 \\
Hip circumference, cm & $86 \pm 9$ & $78 \pm 12$ & 0.05 \\
Waist to height ratio & $0.55 \pm 0.08$ & $0.47 \pm 0.07$ & 0.001 \\
SBP (mmHg) & $119.76 \pm 8.11$ & $106.35 \pm 7.47$ & 0.001 \\
DBP (mmHg) & $74.70 \pm 5.23$ & $65.43 \pm 4.91$ & 0.001 \\
Bone age, years & $15.3 \pm 2.3$ & $12.2 \pm 1.2$ & 0.001 \\
\hline
\end{tabular}

Data are expressed as mean \pm standard deviation

SDS-BMI: standard deviation scores of body mass index, SBP: systolic blood pressure, DBP: diastolic blood pressure, CAH: congenital adrenal hyperplasia, NS non-significant 


\section{Discussion}

This study demonstrates that a) children with classical $\mathrm{CAH}$ may have subclinical LV hypertrophy, diastolic dysfunction and subclinical atherosclerosis; b) EFT was higher in patients with CAH than in the healthy controls; c) EFT is correlated to carotid intima media thickness, LV mass and mitral DcT suggesting that EFT may be used as an additional marker of endothelial and myocardial dysfunction in children with $\mathrm{CAH}$. The classical cardiovascular risk factors in children with $\mathrm{CAH}$, namely obesity, hypertension, dyslipidemia, steroid treatment and others have been extensively discussed elsewhere $(4,5,6)$.
To our knowledge, this study is the first to demonstrate that EFT was significantly increased in children with classical CAH compared with control children $(p<0.001)$. In addition, we showed that EFT was correlated positively with CA-IMT. The multiple linear regression analysis showed that the CA-IMT was the variable that most influenced EFT. EFT was reported to be increased in children with a positive family history of type $2 \mathrm{DM}$ and has been suggested as a risk factor for early atherosclerosis (23). A meta-analysis showed that EFT may be an effective marker for the prediction of coronary heart disease (24). Epicardial fat is thought to play a pivotal role in the pathogenesis of coronary artery disease (CAD)

\begin{tabular}{|c|c|c|c|}
\hline & CAH cases $(n=36)$ & Controls $(n=36)$ & $p$ value \\
\hline Total cholesterol (mg/dL) & $173.65 \pm 43.34$ & $142.22 \pm 18.14$ & 0.01 \\
\hline Triglycerides (mg/dL) & $138.22 \pm 34.23$ & $104.23 \pm 12.21$ & 0.01 \\
\hline LDL-C (mg/dL) & $113.55 \pm 65.21$ & $73.66 \pm 13.32$ & 0.001 \\
\hline HDL-C (mg/dL) & $44.12 \pm 7.8$ & $53.9 \pm 7.8$ & 0.01 \\
\hline Fasting blood glucose (mg/dL) & $92.4 \pm 15.8$ & $81.6 \pm 12.9$ & 0.01 \\
\hline Fasting insulin (lU/mL) & $15.2 \pm 6.2$ & $7.6 \pm 2.8$ & 0.001 \\
\hline HOMA-IR & $3.21 \pm 1.2$ & $1.8 \pm 0.8$ & 0.001 \\
\hline hsCRP (mg/L) & $329 \pm 20.5$ & $154.9 \pm 16.8$ & 0.001 \\
\hline ACTH (pg/mL) & $102.5 \pm 12.7$ & $26.3 \pm 2.2$ & 0.001 \\
\hline 17-OHP (nmol/L) & $184.2 \pm 54.9$ & $1.75 \pm 0.95$ & 0.001 \\
\hline Testosterone (ng/dL) & $544.3 \pm 195.7$ & $181.6 \pm 62.5$ & 0.001 \\
\hline Androstenedione (ng/dL) & $182 \pm 15.2$ & $89.3 \pm 17.2$ & 0.001 \\
\hline
\end{tabular}

Data are expressed as mean \pm standard deviation.

LDL-C: low density lipoprotein cholesterol, HDL-C: high density lipoprotein cholesterol, HOMA-IR: homeostasis model assessment of insulin resistance, ACTH: adrenocorticotropin hormone, 17-OHP: 17-hydroxyprogesterone, hsCRP: high-sensitivity C-reactive protein, CAH: congenital adrenal hyperplasia

Table 3. The echocardiographic, carotid artery intima-media thickness and epicardial fat thickness findings in the congenital adrenal hyperplasia patients compared to controls

\begin{tabular}{|c|c|c|c|}
\hline & CAH children $(n=36)$ & Controls $(n=36)$ & $\mathrm{p}$ value \\
\hline LVEDD (mm) & $43.7 \pm 2.2$ & $37.9 \pm 3.8$ & 0.001 \\
\hline LVESD (mm) & $23.5 \pm 2.1$ & $22.2 \pm 2.8$ & NS \\
\hline $\mathrm{EF}(\%)$ & $67.5 \pm 6.6$ & $66.3 \pm 4.1$ & NS \\
\hline FS (\%) & $41.2 \pm 3.1$ & $49.9 \pm 4.2$ & NS \\
\hline IVSWT (mm) & $9.36 \pm 1.20$ & $4.33 \pm 1.32$ & 0.001 \\
\hline LVPWT (mm) & $7.54 \pm 0.43$ & $4.17 \pm 0.45$ & 0.001 \\
\hline LVMI $\left(\mathrm{gm} / \mathrm{m}^{2}\right)$ & $59.71 \pm 7.24$ & $42.29 \pm 5.75$ & 0.001 \\
\hline E/A ratio & $1.28 \pm 0.12$ & $1.64 \pm 0.22$ & 0.01 \\
\hline IVRT (ms) & $76.2 \pm 6.3$ & $46.5 \pm 5.1$ & 0.001 \\
\hline Mitral DcT (ms) & $189.5 \pm 16.2$ & $129.4 \pm 15.3$ & 0.001 \\
\hline CA-IMT; mm & $0.52 \pm 0.20$ & $0.43 \pm 0.02$ & 0.001 \\
\hline EFT (mm) & $6.95 \pm 0.81$ & $4.01 \pm 0.52$ & 0.001 \\
\hline
\end{tabular}

Data are expressed as mean \pm standard deviation.

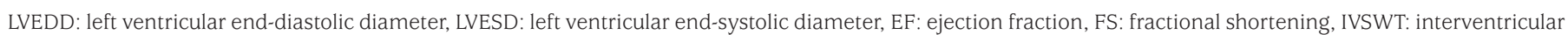

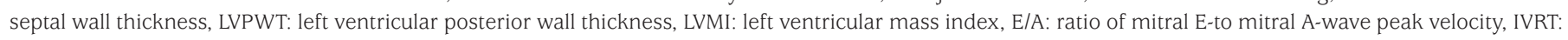
isovolumic relaxation time, DcT: deceleration time, EFT: epicardial fat thickness, NS: non-significant, CA-IMT: carotid artery intima-media thickness 
as it releases a wide range of biologically active molecules that modulate vascular smooth-muscle contraction (25). The paracrine effects of these molecules might be attributable to their location being close to the adventitia and extravascular bed (26). Gastaldelli and Basta (27) reported the existence of a link between epicardial fat and hypertension, atherosclerosis and coronary heart disease. Several studies have emphasized the link between EFT and the severity of CAD (28). Epicardial fat has an important role in the inflammatory process within the atherosclerotic plaque (29). Furthermore, it has been shown that epicardial fat products induce increased cell surface expression of adhesion molecules, enhance adhesion of monocytes to coronary artery endothelial cells, and facilitate migration of adherent monocytes (30).

Echocardiographic EFT measurements, provide some advantages when used to assess the cardiometabolic risk. They are objective, quantified, non-invasive, low cost, have routine applicability, avoid exposure to radiation and have a potential for monitoring therapeutic effects. They may also be used as a simple marker for identification of $\mathrm{CAH}$ patients with higher cardiovascular risk who may need further cardiac evaluation (31).

In the present study, children with $\mathrm{CAH}$ had echocardiographic changes indicating the presence of LV hypertrophy, as indicated by increased LVMI. Moreover, our study showed significant positive correlations between EFT and LVMI that remained significant after regression analysis, which suggested a detrimental effect of EFT excess on the myocardium of patients with $\mathrm{CAH}$. This is in agreement with Corradi et al (32) who reported that EFT levels have an important role in LV hypertrophy. Some mechanisms may be suggested to explain this correlation. It could be assumed that the increased visceral fat directly effects LV output and stroke volume to perfuse the increased body mass. Additionally, the biochemical properties of visceral adipose tissue, such as increased IR, high free fatty acids (FFA) levels, and adrenergic activity, could contribute to LV hypertrophy (33).

In the present study, children with $\mathrm{CAH}$ had echocardiographic changes indicating presence of diastolic dysfunction (as evidenced by reduced E/A ratio and prolonged IVRT and mitral DCT). Regression analysis revealed that EFT in $\mathrm{CAH}$ patients correlates with mitral DcT. This is in agreement with the study of Van der Meer (34) which showed that myocardial fat has progressive and harmful effects on LV diastolic function. Diastolic dysfunction has been considered as one of the first echocardiographic abnormalities to appear in patients with atherosclerotic cardiovascular disease with a high rate of release of FFA (35), which encounter no physical barrier or fascia before reaching the cardiomyocytes (36). Therefore, the myocardium receives a double dose of FFA from both the epicardial fat and the systemic circulation. Epicardial fat is a source of several bioactive molecules that might directly influence the myocardium (37). In metabolic and cardiovascular disease states, these fat tissues expand, becoming hypoxic and dysfunctional and recruiting phagocytic cells which would lead to a reduction in the production of protective cytokines and, eventually, impaired cardiac function $(38,39)$.

BMI and waist circumference are widely accepted measures of generalized adiposity. However they are poor indicators for visceral obesity. It is well known that visceral adipose tissue

Table 4. The demographic, laboratory and echocardiographic characteristics of patients, according to the degree of control on medical treatment

\begin{tabular}{llll}
\hline & Uncontrolled patients $(\mathbf{n}=\mathbf{2 0})$ & Controlled patients $(\mathrm{n}=\mathbf{1 6})$ & $\mathrm{p}$ value \\
\hline Age (years) & $16.2 \pm 0.8$ & $13.1 \pm 2.5$ & 0.01 \\
Bone age (years) & $17.1 \pm 1.2$ & $14.3 \pm 2.2$ & 0.01 \\
HOMA-IR & $4.42 \pm 1.9$ & $2.7 \pm 1.9$ & 0.001 \\
hsCRP (mg/L) & $452 \pm 33.1$ & $216.4 \pm 14.6$ & 0.001 \\
17-OHP (nmol/L) & $188.3 \pm 32.5$ & $8.21 \pm 1.3$ & 0.001 \\
Testosterone (ng/dL) & $498.8 \pm 191.2$ & $34.5 \pm 12.7$ & 0.001 \\
LVMI (gm/m²) & $44.4 \pm 6.5$ & $32.4 \pm 7.2$ & 0.01 \\
EFT (mm) & $8.95 \pm 1.21$ & $6.66 \pm 1.76$ & 0.001 \\
DCT (milliseconds) & $187.0 \pm 23.0$ & $120.0 \pm 25.0$ & 0.01 \\
CA-IMT (mm) & $0.54 \pm 0.30$ & $0.43 \pm 0.02$ & 0.05 \\
\hline
\end{tabular}

Data are expressed as mean \pm standard deviation.

HOMA-IR: homeostasis model assessment for insulin resistance, EFT: epicardial fat thickness, 17-OHP: 17-hydroxyprogesterone, hsCRP: high-sensitivity C-reactive protein, LVMI: left ventricular mass index, DcT: deceleration time, CA-IMT: carotid artery intima-media thickness 
Table 5. The correlation between epicardial fat thickness and anthropometric, laboratory and echocardiographic data in children with congenital adrenal hyperplasia

\begin{tabular}{|c|c|}
\hline Parameter & ( $\mathrm{r}$ and $\mathrm{p}$ values) \\
\hline Age (years) & $+0.639 *$ \\
\hline SDS-BMI & 0.155 \\
\hline Waist circumference $(\mathrm{cm})$ & $+0.569 * *$ \\
\hline Waist to height ratio & $+0.657^{*}$ \\
\hline SBP (mmHg) & $+0.432 * *$ \\
\hline DBP (mmHg) & $+0.361 * *$ \\
\hline HOMA-IR & $+0.562 * *$ \\
\hline hsCRP (mg/L) & $+0.389 *$ * \\
\hline Total cholesterol (mg/dL) & 0.062 \\
\hline Triglycerides (mg/dL) & 0.155 \\
\hline HDL-C (mg/dL) & $-0.658 *$ \\
\hline 17-OHP (nmol/L) & $+0.743 * *$ \\
\hline Testosterone (ng/dL) & $+0.659 * *$ \\
\hline LVMI $\left(g m / m^{2}\right)$ & $+0.301 *$ \\
\hline Mitral DcT (ms) & $+0.39 * *$ \\
\hline CA-IMT (mm) & $+0.415^{* *}$ \\
\hline \multicolumn{2}{|l|}{ "Indicates $p<0.05$} \\
\hline \multicolumn{2}{|c|}{$* *$ Indicates $p<0.01$} \\
\hline \multicolumn{2}{|c|}{$\begin{array}{l}\text { Statistical significance is indicated by asterisks after the correlation } \\
\text { coefficient. }\end{array}$} \\
\hline \multicolumn{2}{|c|}{$\begin{array}{l}\text { SDS-BMI: standard deviation scores of body mass index, LVMI: left ventricular } \\
\text { mass index, SBP: systolic blood pressure, DBP: diastolic blood pressure, } \\
\text { hsCRP: high-sensitivity C-reactive protein, HOMA-IR: the homeostasis model } \\
\text { assessment of insulin resistance, HDL-C: high density lipoprotein cholesterol, } \\
\text { EFT: Epicardial fat thickness, mitral DcT: mitral deceleration time, CA-IMT: } \\
\text { carotid intima media thickness }\end{array}$} \\
\hline
\end{tabular}

Table 6. Multivariate correlation coefficients between epicardial fat thickness and various confounding variables in children with congenital adrenal hyperplasia

\begin{tabular}{lll}
\hline Confounding variables & Odds ratio & $\mathbf{9 5 \% ~ C I}$ \\
\hline Waist circumference $(\mathrm{cm})$ & $1.9^{* *}$ & $1.45-2.4$ \\
$17-O H P(\mathrm{nmol} / \mathrm{L})$ & $1.6^{*}$ & $1.33-2.89$ \\
Testosterone $(\mathrm{ng} / \mathrm{dL})$ & $1.7^{* *}$ & $1.55-2.13$ \\
HOMA-IR & $1.3^{*}$ & $1.04-1.34$ \\
LVMI $\left(\mathrm{gm} / \mathrm{m}^{2}\right)$ & $1.1^{* *}$ & $1.08-1.13$ \\
Mitral DcT $(\mathrm{ms})$ & $1.4^{* *}$ & $1.15-2.05$ \\
CA-IMT $(\mathrm{mm})$ & $2.7^{* *}$ & $1.16-1.57$ \\
\hline
\end{tabular}

Statistical significance is indicated by asterisks after the odds ratio.

${ }^{*}$ Indicates $\mathrm{p}<0.05$.

* * Indicates $p<0.01$

17-OHP: 17-hydroxyprogesterone, EFT: epicardial fat thickness, HOMAIR: the homeostasis model assessment of insulin resistance, LVMI: left ventricular mass index, mitral DcT: mitral deceleration time, CA-IMT: carotid intima media thickness, CI: confidence interval accumulation is associated with subclinical atherosclerosis and increased cardiovascular mortality and morbidity. In this study, we found a very good correlation between EFT and waist circumference by multiple regression analysis in children with $\mathrm{CAH}$. However we did not find a significant correlation between BMI-SDS and EFT. These findings suggest that waist circumference is a better anthropometric cardiovascular risk predictor and support the evidence that EFT is related to visceral fat, rather than total adiposity (40). Mavri et al (41) suggested that CA-IMT regression may also be achieved by weight reduction programs. Altin et al (42) suggested that laparoscopic sleeve gastrectomy induced weight loss results in regression of CA-IMT and EFT. Marked adipose mass reduction is associated with dramatic changes in circulating adipokine levels, with leptin reduction and adiponectin increase, thereby leading to a reduced leptin/ adiponectin ratio. Of note, such a ratio was found to be directly correlated with CA-IMT in male subjects (43).

Testosterone concentrations were significantly higher in our subjects with CAH compared to controls, particularly in children who were poorly controlled on medical treatment. In addition, testosterone correlated positively and significantly with EFT. Colgecen et al (44) reported that subjects in advanced stages of androgenetic alopecia had higher echocardiographically measured EFT than controls. Moreover, Cakir et al (45) reported a strong positive correlation between testosterone concentrations and EFT in patients with polycystic ovarian disease. This finding suggests that androgen excess may be responsible for the increased EFT in patients with CAH. Physicians treating these patients should be aware that amelioration of androgen excess in patients with $\mathrm{CAH}$ should also be considered as a way to prevent cardiovascular symptoms and not only as a tool to improve hyperandrogenic symptoms.

In the present study, children with CAH had higher HOMAIR than controls. EFT correlated positively and significantly with HOMA-IR by multiple regression analysis. This finding is in agreement with Manco et al (46) who reported that epicardial fat is a significant marker of increased insulin resistance. These observations suggest that epicardial fat is a tissue with high IR (47). EFT is associated with high lipolytic activity, probably because of the reduced antilipolytic effect of insulin in this tissue and an increased expression of B-adrenergic receptors, especially B-3 receptors. Stimulation by these receptors activates lipolysis and increases the release of FFAs which are able to promote blood pressure increase through different pathways, including adrenergic stimulation, increased oxidative stress, endothelial dysfunction and vascular cell growth (48). 


\section{Study Limitations}

We recognize that this study has some limitations such us; Due to the difficulties in enrolling and studying $\mathrm{CAH}$ children, the sample was relatively small in size and included patients with a wide age range. Due to the cross-sectional design, it is difficult to generalize the results to the general population. CA-IMT measurements were performed manually. We were not able to confirm EFT using the standard magnetic resonance imaging methods. However, echocardiographic calculation of epicardial fat has been reported to show good reliability when compared with magnetic resonance epicardial fat measurements (49). Epicardial adipose tissue has a threedimensional distribution. Therefore 2D echocardiography may not accurately reflect the total amount of epicardial adiposity.

\section{Conclusions}

EFT is increased in children with classic CAH, particularly in those with poor control and is correlated with CA-IMT, LV mass and mitral DCT. Measurement of EFT by echocardiography in $\mathrm{CAH}$ children may help to identify those at high risk of developing LV dysfunction and subclinical atherosclerosis. Future prospective and multicenter studies are required to confirm our results.

\section{Ethics}

Ethics Committee Approval: The study protocol was approved by the Local Ethics Committee of Assiut University Children Hospital, Assiut, Egypt (approval number: $312 / 2017)$.

Informed Consent: Written informed consent was obtained from the parents of all participants.

Peer-review: Externally peer-reviewed.

\section{Authorship Contributions}

Surgical and Medical Practices: Kotb Abbass Mewalley, Hekma Saad Farghaly, Concept: Kotb Abbass Mewalley, Hekma Saad Farghaly, Design: Kotb Abbass Mewalley, Hekma Saad Farghaly, Data Collection or Processing: Kotb Abbass Mewalley, Hekma Saad Farghaly, Analysis or Interpretation: Kotb Abbass Mewalley, Hekma Saad Farghaly, Abdelrahman Abdelhamid, Literature Search: Kotb Abbass Mewalley, Hekma Saad Farghaly, Writing: Kotb Abbass Mewalley, Hekma Saad Farghaly.

Financial Disclosure: The authors declared that this study received no financial support.

\section{References}

1. Speiser PW, Azziz R, Baskin LS, Ghizzoni L, Hensle TW, Merke DP, Meyer-Bahlburg HF, Miller WL, Montori VM, Oberfield SE, Ritzen M, White PC; Endocrine Society. Congenital adrenal hyperplasia due to steroid 21-hydroxylase deficiency: an Endocrine Society clinical practice guideline. J Clin Endocrinol Metab 2010;95:4133-4160.

2. White PC, Speiser PW. Congenital adrenal hyperplasia due to 21-hydroxylase deficiency. Endocr Rev 2000;21:245-291.

3. Speiser PW, White PC. Congenital adrenal hyperplasia. N Engl J Med 2003;349:776-788.

4. Amr NH, Ahmed AY, Ibrahim YA. Carotid intima media thickness and other cardiovascular risk factors in children with congenital adrenal hyperplasia. J Endocrinol Invest 2014;37:1001-1008. Epub 2014 Aug 12

5. Marra AM, Improda N, Capalbo D, Salzano A, Arcopinto M, De Paulis A, Alessio M, Lenzi A, Isidori AM, Cittadini A, Salerno M. Cardiovascular abnormalities and impaired exercise performance in adolescents with congenital adrenal hyperplasia. J Clin Endocrinol Metab 2015;100:644652. Epub 2014 Nov 18

6. Mooij CF, Kroese JM, Claahsen-van der Grinten HL, Tack CJ, Hermus AR. Unfavourable trends in cardiovascular and metabolic risk in paediatric and adult patients with congenital adrenal hyperplasia? Clin Endocrinol(Oxf) 2010;73:137-146. Epub 2009 Aug 29

7. Wasniewska M, Balsamo A, Valenzise M, Manganaro A, Faggioli G, Bombaci S, Conti V, Ferri M, Aversa T, Cicognani A, De Luca F. Increased large artery intima media thickness in adolescents with either classical or non-classical congenital adrenal hyperplasia. J Endocrinol Investig 2013;36:12-15. Epub 2011 Dec 21

8. Fusaro MF, Zanini JL, Silva IN. Increased carotid intima-media thickness in Brazilian adolescents with type 1 diabetes mellitus. Diabetol Metab Syndr 2016;8:74.

9. Iacobellis G, Assael F, Ribaudo MC, Zappaterreno A, Alessi G, Di Mario U, Leonetti F. Epicardial fat from echocardiography: a new method for visceral adipose tissue prediction. Obes Res 2003;11:304-310

10. Bachar GN, Dicker D, Kornowski R, Atar E. Epicardial adipose tissue as a predictor of coronary artery disease in asymptomatic subjects. Am J Cardiol 2012;110:534-538. Epub 2012 May 10

11. Merke DP, Bornstein SR. Congenital adrenal hyperplasia. Lancet 2005;365:2125-2136

12. Labarta JI, Bello E, Ruiz-Echarri M, Rueda C, Martul P, Mayayo E, Ferrández Longás A. Childhood-onset congenital adrenal hyperplasia: long-term outcome and optimization of therapy. J Pediatr Endocrinol Metab 2004;17(Suppl 3):411-422.

13. Joint LWPES/ESPE CAH Working Group. Consensus statement on 21-hydroxylase deficiency from the Lawson Wilkins Pediatric Endocrine Society and the European Society for Paediatric Endocrinology. J Clin Endocrinol Metab 2002;87:4048-4053.

14. Finkielstain GP, Kim MS, Sinaii N, Nishitani M, Van Ryzin C, Hill SC, Reynolds JC, Hanna RM, Merke DP. Clinical characteristics of a cohort of 244 patients with congenital adrenal hyperplasia. J Clin Endocrinol Metab 2012;97:4429-4438. Epub 2012 Sep 18

15. Diabetes Endocrine Metabolism Pediatric Unit, Cairo University Children's Hospital. Egyptian growth curves 2002. [http://dempuegypt. blogspot.com]

16. Tanner JM, Whitehouse RH. A note on the bone age at which patients with true isolated growth hormone deficiency enter puberty. J Clin Endocrinol Metab 1975;41:788-790

17. Greulich WW, Pyle SI, Stanford CA. Radiographic Atlas of Skeletal Development of the Hand and Wrist. Calif Med 1959;91:53. 
18. Bretones P, Riche B, Pichot E, David M, Roy P, Tardy V, Kassai B, Gaillard S, Bernoux D, Morel Y, Chatelain P, Nicolino M, Cornu C; French Collaborative CAH Growth Study Group. Growth curves for congenital adrenal hyperplasia from a national retrospective cohort. J Pediatr Endocrinol Metab 2016;29:1379-1388.

19. Chen Y, Zhang X, Pan B, Jin X, Yao H, Chen B, Zou Y, Ge J, Chen H. A modified formula for calculating low-density lipoprotein cholesterol values. Lipids Health Dis 2010;9:52.

20. Gutch M, Kumar S, Razi SM, Gupta KK, Gupta A. Assessment of insulin sensitivity/resistance. Indian J Endocrinol Metab 2015;19:160-164.

21. Park MK. Pediatric cardiology for practitioners. Noninvasive techniques (Chap. 6), 5th ed. An imprint of Elsevier, Part2: Special tools in evaluation of cardiac patients Elsevier, Mosby, 2008.

22. Järvisalo MJ, Jartti L, Näntö-Salonen K, Irjala K, Rönnemaa T, Hartiala JJ, Celermajer DS, Raitakari OT. Increased aortic intima-media thickness: a marker of preclinical atherosclerosis in high-risk children. Circulation 2001;104:2943-2947.

23. Mahfouz RA, Alzaiat A, Yousry A. Relationship of epicardial fat thickness with endothelial and cardiac functions in children with family history of type 2 diabetes mellitus. Echocardiography 2015;32:28-33. Epub 2014 Mar 25

24. Baker AR, Silva NF, Quinn DW, Harte AL, Pagano D, Bonser RS, Kumar S, McTernan PG. Human epicardial adipose tissue expresses a pathogenic profile of adipocytokines in patients with cardiovascular disease. Cardiovasc Diabetol 2006;5:1.

25. Bachar GN, Dicker D, Kornowski R, Atar E. Epicardial adipose tissue as a predictor of coronary artery disease in asymptomatic subjects. Am J Cardiol 2012;110:534-538. Epub 2012 May 10

26. Sengul C, Cevik C, Ozveren O, Duman D, Eroglu E, Oduncu V, Tanboga HI, Can MM, Akgun T, Dindar I. Epicardial fat thickness is associated with non-dipper blood pressure pattern in patients with essential hypertension. Clin Exp Hypertens 2012;34:165-170. Epub 2011 Oct 18

27. Gastaldelli A, Basta G. Ectopic fat and cardiovascular disease: what is the link? Nutr Metab Cardiovasc Dis 2010;20:481-490. Epub 2010 Jul 24

28. Nakanishi R, Rajani R, Cheng VY, Gransar H, Nakazato R, Shmilovich H, Otaki Y, Hayes SW, Thomson LE, Friedman JD, Slomka PJ, Berman DS, Dey D. Increase in epicardial fat volume is associated with greater coronary artery calcification progression in subjects at intermediate risk by coronary calcium score: a serial study using non-contrast cardiac CT. Atherosclerosis 2011;218:363-368. Epub 2011 Jul 23

29. Şengül C, Özveren O. Epicardial adipose tissue: a review of physiology, pathophysiology, and clinical applications. Anadolu Kardiyol Derg 2013;13:261-265. Epub 2013 Feb 6

30. Cengel A. Epicardial adipose tissue, metabolic syndrome, inflammation, and cardiovascular risk. Turk Kardiyol Dern Ars 2012;40:696-698.

31. Iacobellis G, Willens HJ. Echocardiographic epicardial fat: a review of research and clinical applications. J Am Soc Echocardiogr 2009;22:13111319 .

32. Corradi D, Maestri R, Callegari S, Pastori P, Goldoni M, Luong TV, Bordi $\mathrm{C}$. The ventricular epicardial fat is related to the myocardial mass in normal, ischemic and hypertrophic hearts. Cardiovasc Pathol 2004;13:313-316.

33. Iacobellis G, Ribaudo MC, Zappaterreno A, Iannucci CV, Leonetti F. Relation between epicardial adipose tissue and left ventricular mass. Am J Cardiol 2004;94:1084-1087.

34. van der Meer RW, Rijzewijk LJ, Diamant M, Hammer S, Schär M, Bax JJ, Smit JW, Romijn JA, de Roos A, Lamb HJ. The ageing male heart: myocardial triglyceride content as independent predictor of diastolic function. Eur Heart J 2008;29:1516-1522. Epub 2008 May 19

35. Harada M, Tabako S. Carotid atherosclerosis is associated with left ventricular diastolic function. J Echocardiogr 2016;14:120-129. Epub 2016 Jun 30

36. Iacobellis G, Corradi D, Sharma AM. Epicardial adipose tissue: anatomic, biomolecular and clinical relationships with the heart. Clin Pract Cardiovasc Med 2005;2:536-543.

37. Greenstein AS, Khavandi K, Withers SB, Sonoyama K, Clancy O, Jeziorska M, Laing I, Yates AP, Pemberton PW, Malik RA, Heagerty AM. Local inflammation and hypoxia abolish the protective anticontractile properties of perivascular fat in obese patients. Circulation 2009;119:1661-1670. Epub 2009 Mar 16

38. Henrichot E, Juge-Aubry CE, Pernin A, Pache JC, Velebit V, Dayer JM, Meda P, Chizzolini C, Meier CA. Production of chemokines by perivascular adipose tissue: a role in the pathogenesis of atherosclerosis? Arterioscler Thromb Vasc Biol 2005;25:2594-2599. Epub 2005 Sep 29

39. Xu Y, Cheng X, Hong K, Huang C, Wan L. How to interpret epicardial adipose tissue as a cause of coronary artery disease: a meta-analysis. Coron Artery Dis 2012;23:227-233.

40. Janssen I, Heymsfield SB, Allison DB, Kotler DP, Ross R. Body mass index and waist circumference independently contribute to the prediction of nonabdominal, abdominal subcutaneous, and visceral fat. Am J Clin Nutr 2002;75:683-688.

41. Mavri A, Stegnar M, Sentocnik JT, Videcnik V. Impact of weight reduction on early carotid atherosclerosis in obese premenopausal women. Obes Res 2001;9:511-516.

42. Altin C, Erol V, Aydin E, Yilmaz M, Tekindal MA, Sade LE, Gulay H, Muderrisoglu $\mathrm{H}$. Impact of weight loss on epicardial fat and carotid intima media thickness after laparoscopic sleeve gastrectomy: A prospective study. Nutr Metab Cardiovasc Dis 2018;28:501-509. Epub 2018 Feb 9

43. Norata GD, Raselli S, Grigore L, Garlaschelli K, Dozio E, Magni P, Catapano AL. Leptin: adiponectin ratio is an independent predictor of intima media thickness of the common carotid artery. Stroke 2007;38:2844-2846. Epub 2007 Sep 6

44. Colgecen E, Ede H, Erkoc MF, Akyuz Y, Erbay AR. The Relation of Androgenetic Alopecia Severity with Epicardial Fat Thickness. Ann Dermatol 2016;28:205-209. Epub 2016 Mar 31

45. Cakir E, Doğan M, Topaloglu O, Ozbek M, Cakal E, Vural MG, Yeter E, Delibasi T. Subclinical atherosclerosis and hyperandrogenemia are independent risk factors for increased epicardial fat thickness in patients with PCOS and idiopathic hirsutism. Atherosclerosis 2013;226:291-295. Epub 2012 Nov 15

46. Manco M, Morandi A, Marigliano M, Rigotti F, Manfredi R, Maffeis C. Epicardial fat, abdominal adiposity and insulin resistance in obese prepubertal and early pubertal children. Atherosclerosis 2013;226:490495. Epub 2012 Dec 3

47. Marchington JM, Pond CM. Site specific properties of pericardial and epicardial adipose tissue: the effects of insulin and high-fat feeding on lipogenesis and the incorporation of fatty acids in vivo. Int J Obes 1990;14:1013-1022.

48. Fortier M, Wang SP, Mauriège P, Semache M, Mfuma L, Li H, Levy E, Richard D, Mitchell GA. Hormone-sensitive lipase-independent adipocyte lipolysis during $\beta$-adrenergic stimulation, fasting, and dietary fat loading. Am J Physiol Endocrinol Metab 2004;287:282-288.

49. Iacobellis G, Assael F, Ribaudo MC, Zappaterreno A, Alessi G, Di Mario U, Leonetti F. Epicardial fat from echocardiography: a new method for visceral adipose tissue prediction. Obes Res 2003;11:304-310. 